\title{
A Note on Modified Estimators for the Parameters of the Power Function Distribution
}

\author{
Azam Zaka ${ }^{1}$, Navid Feroze ${ }^{2}$, and Ahmad Saeed Akhter ${ }^{3}$ \\ ${ }^{1}$ College of Statistical and Actuarial Sciences, University of the Punjab, Lahore, \\ Pakistan \\ ${ }^{2}$ Department of Statistics, Government Post Graduate College Muzaffarabad, Azad \\ Kashmir, Pakistan \\ ${ }^{3}$ College of Statistical and Actuarial Sciences, University of the Punjab, Lahore, \\ Pakistan \\ ${ }^{1}$ logic_azam@hotmail.com,azamzka@gmail.com, \\ 2navidferoz@hotmail.com, ${ }^{3}$ akhtar@stat.pu.edu.pk
}

\begin{abstract}
This paper is concerned with the modifications of maximum likelihood, moments and percentile estimators of the two parameter Power function distribution. Sampling behavior of the estimators is indicated by Monte Carlo simulation. For some combinations of parameter values, some of the modified estimators appear better than the traditional maximum likelihood, moments and percentile estimators with respect to bias, mean square error and total deviation.
\end{abstract}

Keywords: percentile estimators, maximum likelihood estimators, moment estimators, modified estimators, total deviation, mean square error

\section{Introduction}

The Power function distribution is a flexible life time distribution model that may offer a good fit to some sets of failure data. Theoretically, Power function distribution is a special case of Pareto distribution. An excellent account of this distribution and its properties is given in Kleiber and Kotz [8]. Meniconi and Barry [15] discussed the application of Power function distribution. They proved that the Power function distribution is the best distribution to check the reliability of any electrical component. They used Exponential distribution, Lognornal distribution and Weibull distribution and showed from reliability and hazard function that Power function distribution is the best distribution.

The probability distribution of Power function distribution is

$$
\mathrm{f}(\mathrm{t})=\frac{\gamma \mathrm{t}^{\gamma-1}}{\beta^{\gamma}} ; \quad 0<\mathrm{t}<\beta
$$

With shape parameter $\gamma$ and scale parameter $\beta$, the interval $(0, \beta)$

Rider [20] derived distributions of the product and quotients of the order statistics from a Power function distribution. Moments of order statistics for a Power function distribution were calculated by Malik [13]. Dubey [7] discussed the percentile estimators for Weibull parameters. Lwin [12], Arnold and Press [19] discussed Bayesian estimation for the scale parameter of the Pareto distribution using a Power function prior. Ahsanullah [1] discussed 
the estimation of the location and scale parameters of a Power function distribution. Kapadia [9] discussed the sample size required to estimate a parameter in the Power function distribution.

Cohen and Whitten [4-6] used the moment and modified moment estimators for the Weibull distribution. Samia and Mohamed [23] used five modifications of moments to estimate the parameters of the Pareto distribution. Lalitha and Anand [11] used modified maximum likelihood to estimate the scale parameter of the Rayleigh distribution. Rafiq et al., [21] discussed the parameters of the Gamma distribution. Rafiq et al., [22] discussed the method of fractional moments to estimate the parameters of Weibull distribution. Kang and Young [10] estimated the parameters of a Pareto distribution by jackknife and bootstrap methods. Munawar et al., [16] discussed the Bayesian parameters estimation of hybrid censored power function distribution under different loss functions. Marks [17] estimated the parameters of Weibull distribution with the help of percentiles. He called it Common Percentile Method. Zarrin et al., [24] discussed the reliability computation and Bayesian analysis of system reliability of Power function distribution.

In this paper, we use the percentiles method, maximum likelihood and moment method to estimate the two parameters of the Power function distribution. The present paper introduces the modified estimators for parameters of the power function distribution. The resulting estimators are easier to calculate than the maximum likelihood and for certain combinations of parameter values; they improve the estimated values and mean square error. We examined these methods using two parameters Power function distribution to find the most accurate method (the method which has least M.S.E).

\section{Methodology}

This section contains the derivation of the estimators for the parameters of the power function distribution using percentile estimation, maximum likelihood estimation and moment estimation methods. The modifications of the said methods have also been presented.

\subsection{Percentile Estimator (P.E)}

Let $t_{1}, t_{2}, t_{3}, \ldots, t_{n}$ be a random sample of size $n$ drawn from probability density function of Power function distribution. The cumulative distribution function for a Power function distribution with shape and scale parameters $\beta$ and $\gamma$, respectively.

By solving we get

$$
\mathrm{F}\left(\mathrm{t}_{\mathrm{i}}\right)=\left(\frac{\mathrm{t}_{\mathrm{i}}}{\beta}\right)^{\gamma}
$$

$$
t_{i}=\beta\left(R_{i}\right)^{1 / \gamma}
$$

where $R_{i}=F\left(t_{i}\right)$. Let $P_{75}$ and $P_{25}$ are used. Therefore (3)becomes

$$
\begin{aligned}
& \mathrm{P}_{75}=\beta(0.75)^{1 / \gamma} \\
& \mathrm{P}_{25}=\beta(0.25)^{1 / \gamma}
\end{aligned}
$$

Solving (4) and (5) we get

$$
\hat{\gamma}=\frac{\ln \left(\frac{0.75}{0.25}\right)}{\ln \left(\frac{P_{75}}{P_{25}}\right)} \quad \text { and } \quad \hat{\beta}=\frac{P_{75}}{(.75)^{1 / \hat{\gamma}}}
$$


generally $\hat{\gamma}=\frac{\ln \left(\frac{\mathrm{H}}{\mathrm{L}}\right)}{\ln \left(\frac{\mathrm{P}_{\mathrm{H}}}{\mathrm{P}_{\mathrm{L}}}\right)}$

$$
\text { and } \quad \hat{\beta}=\frac{\mathrm{P}_{\mathrm{H}}}{(\mathrm{H})^{1 / \widehat{\gamma}}}
$$

\subsection{First Modified Percentile Estimator (M.P.E.1)}

In this modification of the percentile estimators, eq (5) is replaced by the coefficient of variation of Power function distribution.

$$
\text { c. } v=\frac{1}{\sqrt{\gamma(\gamma+2)}}
$$

and $\quad \mathrm{P}_{75}=\beta(0.75)^{1 / \gamma}$

From (8) $\quad \frac{\mathrm{s}}{\overline{\mathrm{t}}}=\frac{1}{\sqrt{\gamma(\gamma+2)}}$

After simplifications, we get

$$
\hat{\gamma}=-1+\sqrt{1+\frac{\overline{\mathrm{t}}^{2}}{\mathrm{~S}^{2}}}
$$

From (4) $\quad \widehat{\beta}=\frac{P_{75}}{(0.75)^{1 / \gamma}}$

$$
\hat{\beta}=\frac{\mathrm{P}_{75}}{(0.75)^{1 /\left(-1+\sqrt{1+\frac{\overline{\mathrm{t}}^{2}}{\mathrm{~S}^{2}}}\right)}}=>\hat{\beta}=\frac{\mathrm{P}_{\mathrm{H}}}{(\mathrm{H})^{1 /\left(-1+\sqrt{1+\frac{\mathrm{t}^{2}}{\mathrm{~S}^{2}}}\right)}}
$$

\subsection{Second Modified Percentile Estimator (M.P.E.2)}

In this modification of the percentile estimators, eq (5) is replaced by the Median of Power function distribution.

$\tilde{\mathrm{t}}=\frac{\beta}{2^{1 / \gamma}}$

From (4) $\quad \hat{\beta}=\frac{P_{75}}{(0.75)^{1 / \gamma}}$

therefore $\quad \tilde{\mathrm{t}} 2^{1 / \gamma}=\frac{\mathrm{P}_{75}}{(0.75)^{1 / \gamma}}=>\hat{\gamma}=\frac{\ln (2 * 0.75)}{\ln \left(\frac{\mathrm{P}_{75}}{\tilde{\mathfrak{t}}}\right)} \quad=>\hat{\gamma}=\frac{\ln (2 * \mathrm{H})}{\ln \left(\frac{\mathrm{P}_{\mathrm{H}}}{\tilde{\mathrm{t}}}\right)}$

\subsection{Third Modified Percentile Estimator (M.P.E.3)}

Using $\mathrm{E}\left(F\left(\mathrm{t}_{1}\right)\right)=F\left(\mathrm{t}_{1}\right)$ of Power function distribution and (4), by neglecting(5).

$\mathrm{P}_{75}=\beta(0.75)^{1 / \gamma}$

$$
\widehat{\beta}=\frac{P_{75}}{(0.75)^{1 / \gamma}}
$$

And

$$
\mathrm{E}\left(F\left(\mathrm{t}_{1}\right)\right)=F\left(\mathrm{t}_{1}\right)
$$

$$
\frac{1}{\mathrm{n}+1}=\left(\frac{\mathrm{t}_{(1)}}{\beta}\right)^{\gamma}=>\widehat{\beta}=\mathrm{t}_{(1)}(\mathrm{n}+1)^{1 / \gamma}
$$


Solving (13) and (15), we have

$$
\hat{\gamma}=\frac{\ln (0.75 *(\mathrm{n}+1))}{\ln \left(\frac{\mathrm{P}_{75}}{\mathrm{t}_{(1)}}\right)} \quad \Rightarrow \quad \hat{\gamma}=\frac{\ln (\mathrm{H} *(\mathrm{n}+1))}{\ln \left(\frac{\mathrm{P}_{\mathrm{H}}}{\mathrm{t}_{(1)}}\right)}
$$

\subsection{Maximum Likelihood Method (M.L.E)}

Let $t_{1}, t_{2}, t_{3}, \ldots, t_{n}$ be a random sample of size $n$ drawn from probability density function of Power function distribution. The likelihood function of this random sample is the joint density of the $n$ random variables and is a function of the unknown parameters. Thus

$\mathrm{f}(\mathrm{x})=\frac{\gamma \mathrm{t}^{\gamma-1}}{\beta^{\gamma}} \quad 0<t<\beta$

Likelihood function is

$\mathrm{L}(\gamma, \beta)=\prod_{\mathrm{i}=1}^{\mathrm{n}} \mathrm{f}\left(\mathrm{t}_{\mathrm{i}}, \gamma, \beta\right)$

$\mathrm{L}(\gamma, \beta)=\frac{\gamma^{\mathrm{n}} \prod_{\mathrm{i}=0}^{\mathrm{n}} \mathrm{t}^{\gamma-1}}{\beta^{\mathrm{n} \gamma}}$

taking natural log on both sides we get

$\ln L=n \ln \gamma+\sum_{\mathrm{i}=1}^{\mathrm{n}} \ln \mathrm{t}^{\gamma-1}-\mathrm{n} \gamma \ln (\beta)$

The maximum likelihood estimator MLE of the parameter is the value of the parameter that maximizes L and MLM for 2 parameter of Power Function distribution can be obtained by solving the equations resulting from setting the two partial derivatives of $L(\gamma, \beta)$ to zero;

$$
\frac{\partial \ln \mathrm{L}}{\partial \beta}=0+0-\frac{\mathrm{n} \gamma}{\beta}=0
$$

$\beta$ does not exist. but the likelihood function can be maximize by taking

$$
\widehat{\beta}=t_{n}
$$

Where $t_{n}$ is the largest value in the sample data.

$$
\begin{aligned}
& \frac{\partial \ln \mathrm{L}}{\partial \gamma}=\frac{\mathrm{n}}{\gamma}+\sum_{\mathrm{i}=1}^{\mathrm{n}} \ln \mathrm{t}_{\mathrm{i}}-\mathrm{n} \ln (\beta)=0 \\
& \hat{\gamma}=\left(\frac{\mathrm{n}}{\mathrm{n} \ln (\beta)-\sum_{\mathrm{i}=1}^{\mathrm{n}} \ln \mathrm{t}_{\mathrm{i}}}\right)
\end{aligned}
$$

\subsection{First Modified Maximum Likelihood Estimator (M.M.L.E.1)}

By neglecting (17) and replacing

$\mathrm{E}\left(F\left(\mathrm{t}_{1}\right)\right)=F\left(\mathrm{t}_{1}\right)$

$$
\frac{1}{\mathrm{n}+1}=\left(\frac{\mathrm{t}_{(1)}}{\beta}\right)^{\gamma} \quad \Rightarrow \quad \widehat{\beta}=\mathrm{t}_{(1)}(\mathrm{n}+1)^{1 / \gamma}
$$

Put in (19) we get 


$$
\begin{aligned}
& \frac{\mathrm{n}}{\gamma}+\sum_{\mathrm{i}=1}^{\mathrm{n}} \ln \mathrm{t}_{\mathrm{i}}-\mathrm{n} \ln \left(\mathrm{t}_{(1)}(\mathrm{n}+1)^{1 / \gamma}\right)=0 \\
& \text { therefore } \quad \hat{\gamma}=\frac{\{1-\ln (\mathrm{n}+1)\}}{\ln \mathrm{t}_{(1)}-\frac{\sum_{\mathrm{i}=1}^{\mathrm{n}} \ln \mathrm{t}_{\mathrm{i}}}{\mathrm{n}}}
\end{aligned}
$$

\subsection{Second Modified Maximum Likelihood Estimator (M.M.L.E. 2)}

From equation (17) and (19)

$$
\begin{aligned}
& \frac{\mathrm{n} \gamma}{\beta}=0 \\
& \frac{\mathrm{n}}{\gamma}+\sum_{\mathrm{i}=1}^{\mathrm{n}} \ln \mathrm{t}_{\mathrm{i}}-\mathrm{n} \ln (\beta)=0
\end{aligned}
$$

Replacing (23) by median of Power function distribution

$$
\tilde{\mathrm{t}}=\frac{\beta}{2^{1 / \gamma}}
$$

i.e.,

$$
\widehat{\beta}=\tilde{\mathrm{t}} 2^{1 / \gamma}
$$

Put in (24)

$$
\begin{array}{r}
\frac{\mathrm{n}}{\gamma}+\sum_{\mathrm{i}=1}^{\mathrm{n}} \ln \mathrm{t}_{\mathrm{i}}-\mathrm{n} \ln \left(\tilde{\mathrm{t}} 2^{1 / \gamma}\right)=0 \\
\hat{\gamma}=\left[\frac{\{1-\ln (2)\}}{\ln (\tilde{\mathfrak{t}})-\frac{\sum_{\mathrm{i}=1}^{\mathrm{n}} \ln \mathrm{t}_{\mathrm{i}}}{\mathrm{n}}}\right]
\end{array}
$$

\subsection{Third Modified Maximum Likelihood Estimator (M.M.L.E.3)}

Using c.v of power function distribution and neglecting $\frac{\partial \ln L}{\partial \beta}$

$$
\begin{aligned}
& \frac{\partial \ln \mathrm{L}}{\partial \gamma}=\frac{\mathrm{n}}{\gamma}+\sum_{\mathrm{i}=1}^{\mathrm{n}} \ln \mathrm{t}_{\mathrm{i}}-\mathrm{n} \ln (\beta)=0 \\
& \text { c. } \mathrm{v}=\frac{1}{\sqrt{\gamma(\gamma+2)}}
\end{aligned}
$$

$\frac{\mathrm{S}}{\overline{\mathrm{X}}}=\frac{1}{\sqrt{\gamma(\gamma+2)}}$

by solving we get $\quad \hat{\gamma}=-1+\sqrt{1+\frac{\overline{\mathrm{t}}^{2}}{\mathrm{~S}^{2}}}$

and $\frac{\partial \ln L}{\partial \gamma}=\frac{n}{\gamma}+\sum_{i=1}^{n} \ln t_{i}-n \ln (\beta)=0$

$$
\widehat{\beta}=e^{\left\{\frac{\sum_{i=1}^{n} \ln t_{i}}{n}+\frac{1}{\gamma}\right\}}
$$




\subsection{Moment Estimators (M.E)}

The method of moments is another technique commonly used in the field of estimation of parameters. If the numbers $t_{1}, t_{2}, t_{3}, \ldots, t_{n}$ represent a set of data, then an unbiased estimator for the $\mathrm{k}^{\text {th }}$ origin moment is

$\mathrm{m}_{\mathrm{k}}^{\prime}=\frac{\sum_{\mathrm{i}=1}^{\mathrm{n}} \mathrm{t}_{\mathrm{i}}^{\mathrm{k}}}{\mathrm{n}}$

Where $\mathrm{m}_{\mathrm{k}}$ stands for thr $\mathrm{k}^{\text {th }}$ sample moment.

The first moment of power function distribution is

$\mathrm{E}(\mathrm{t})=\int_{-\infty}^{\infty} \mathrm{t} f(\mathrm{t}) \mathrm{dt}=>\mathrm{E}(\mathrm{t})=\int_{0}^{\beta} \mathrm{t}\left(\frac{\gamma \mathrm{t}^{\gamma-1}}{\beta^{\gamma}}\right) \mathrm{dt}=\frac{\beta \gamma}{\gamma+1}$

And $\quad E\left(t^{2}\right)=\int_{0}^{\beta} t^{2}\left(\frac{\gamma t^{\gamma-1}}{\beta^{\gamma}}\right) d t=\frac{\beta^{2} \gamma}{\gamma+2}$

Therefore by equating sample and population moments we get

$$
\mathrm{m}_{1}^{\prime}=\mu_{1}^{\prime}=\mathrm{E}(\mathrm{t})=\frac{\beta \gamma}{\gamma+1}
$$

And $\quad \mathrm{m}_{2}^{\prime}=\mu_{2}^{\prime}=\mathrm{E}\left(\mathrm{t}^{2}\right)=\frac{\beta^{2} \gamma}{\gamma+2}$

From (30) $\quad \overline{\mathrm{t}}=\frac{\beta \gamma}{\gamma+1}$

$\overline{\mathrm{t}}(\gamma+1)=\beta \gamma$

$$
\widehat{\beta}=\frac{\overline{\mathrm{t}}(\gamma+1)}{\gamma}
$$

Put in (32), we get

$\mathrm{m}_{2}=\frac{\overline{\mathrm{t}}^{2}(\gamma+1)^{2}}{\gamma(\gamma+2)}$

we know that $\quad \mathrm{m}_{2}=\mathrm{m}_{2}^{\prime}-\left(\mathrm{m}_{1}^{\prime}\right)^{2}$

$\mathrm{m}_{2}^{\prime}=\mathrm{S}^{2}+\overline{\mathrm{t}}^{2} \quad$; where $\mathrm{m}_{2}=\mathrm{S}^{2}$ and $\mathrm{m}_{1}^{\prime}=\overline{\mathrm{t}}$

therefore $\mathrm{S}^{2}+\overline{\mathrm{t}}^{2}=\frac{\overline{\mathrm{t}}^{2}(\gamma+1)^{2}}{\gamma(\gamma+2)}$

Hence $\hat{\gamma}=-1+\sqrt{1+\frac{\overline{\mathrm{t}}^{2}}{\mathrm{~S}^{2}}}$

\subsection{First Modified Moment Estimator (M.M.E.1)}

In this modification of the moment estimators, the second moment of two parameters Power function distribution is replaced by the coefficient of variation of Power function distribution.

$$
\text { C. } v=\frac{1}{\sqrt{\gamma(\gamma+2)}}
$$

and $\mathrm{m}_{1}^{\prime}=\frac{\beta \gamma}{\gamma+1}$ 
$\overline{\overline{\mathrm{t}}}=\frac{1}{\sqrt{\gamma(\gamma+2)}}$

After simplifications, we get

$$
\hat{\gamma}=-1+\sqrt{1+\frac{\overline{\mathrm{t}}^{2}}{\mathrm{~S}^{2}}}
$$

\subsection{Second Modified Moment Estimator (M.M.E.2)}

In this modification of the moment estimators, the second moment of two parameters Power function distribution is replaced by the variance of Power function distribution.

i.e., $\mathrm{S}^{2}=\frac{\beta^{2} \gamma}{(\gamma+2)(\gamma+1)^{2}}$

and $\quad \mathrm{m}_{1}^{\prime}=\frac{\beta \gamma}{\gamma+1}$

From (37) $\quad \hat{\beta}=\frac{s^{2}(\gamma+1)(\gamma+2)}{\overline{\mathrm{t}}} \quad$ put in (31) $\quad \mathrm{m}_{1}^{\prime}=\frac{\mathrm{s}^{2}(\gamma+1)(\gamma+2)}{\overline{\mathrm{t}}} * \frac{\gamma}{\gamma+1}$

$\gamma^{2}+2 \gamma-\frac{\mathrm{m}_{1}^{2}}{\mathrm{~S}^{2}}=0$

where $\quad \mathrm{m}_{1}^{\prime}=\overline{\mathrm{t}}$ therefore, $\quad \gamma^{2}+2 \gamma-\frac{\overline{\mathrm{t}}^{2}}{\mathrm{~s}^{2}}=0$

$$
\hat{\gamma}=-1+\sqrt{1+\frac{\overline{\mathrm{t}}^{2}}{\mathrm{~S}^{2}}}
$$

and $\widehat{\beta}=\frac{\mathrm{S}^{2}(\hat{\gamma}+1)(\hat{\gamma}+2)}{\overline{\mathrm{t}}}$

\subsection{Third Modified Moment Estimator (M.M.E.3)}

In this modification of the moment estimators, the first moment of two parameters Power function distribution is replaced by the variance of Power function distribution.

$$
\begin{array}{ll}
\text { i.e., } & \mathrm{S}^{2}=\frac{\beta^{2} \gamma}{(\gamma+2)(\gamma+1)^{2}} \\
\text { and } & \mathrm{m}_{2}^{\prime}=\frac{\beta^{2} \gamma}{\gamma+2} \\
\text { from (37) } & \beta^{2}=\frac{(\gamma+2)(\gamma+1)^{2} S^{2}}{\gamma}
\end{array}
$$

$$
\begin{array}{ll}
\hat{\beta}=\mathrm{S}(\hat{\gamma}+1) \sqrt{(1+2 / \hat{\gamma})} & \text { putin (32) } \mathrm{m}_{2}^{\prime}=\frac{(\gamma+2)(\gamma+1)^{2} \mathrm{~S}^{2}}{\gamma} * \frac{\gamma}{(\gamma+2)} \\
\mathrm{m}_{2}^{\prime}=(\gamma+1)^{2} \mathrm{~S}^{2} & \\
\hat{\gamma}=-1+\sqrt{1+\frac{\mathrm{t}^{2}}{\mathrm{~S}^{2}}} &
\end{array}
$$

and $\quad \hat{\beta}=S(\hat{\gamma}+1) \sqrt{(1+2 / \hat{\gamma})}$ 


\subsection{Fourth Modified Moment Estimator (M.M.E.4)}

Using co-efficient of variation of Power function distribution and $\mathrm{m}_{2}^{\prime}$, by neglecting $\mathrm{m}_{1}^{\prime}$.

$$
\mathrm{m}_{2}^{\prime}=\frac{\beta^{2} \gamma}{\gamma+2}
$$

C. $\mathrm{v}=\frac{1}{\sqrt{\gamma(\gamma+2)}}$

From (32) $\quad \beta^{2}=\mathrm{m}_{2}^{\prime} * \frac{(\gamma+2)}{\gamma} \quad$ from (35) $\gamma(\gamma+2)=\frac{\overline{\mathrm{t}}^{2}}{\mathrm{~s}^{2}}$, therefore $\hat{\gamma}=-1+$ $\sqrt{1+\frac{\overline{\mathrm{t}}^{2}}{\mathrm{~S}^{2}}}$

and $\quad \hat{\beta}=\sqrt{\frac{(\hat{\gamma}+2) m_{2}^{\prime}}{\hat{\gamma}}}$

Given a sample of $\left(\mathrm{X}_{\mathrm{i}}, \mathrm{Y}_{\mathrm{i}}\right)$ pairs, regression analysis yielding $\hat{\beta}$ and $\hat{\gamma}$ is possible from eq(9). Three approximations for $\hat{F}\left(t_{i}\right)$ based on its being uniformly distributed on interval $[0$, 1] show in Table.

\begin{tabular}{|c|c|c|}
\hline & Method & $\mathrm{F}\left(\mathrm{t}_{\mathrm{i}}\right)$ \\
\hline (a) & Median Rank & $\mathrm{i}-.3$ \\
\hline (b) & Mean Rank & $\overline{\mathrm{n}+.4}$ \\
\hline (c) & Symmetrical Cdf & $\begin{array}{l}n+1 \\
\mathrm{i}-.5\end{array}$ \\
\hline & methods for estimating $\mathrm{F}\left(\mathrm{t}_{\mathrm{i}}\right)$ & $\mathrm{n}$ \\
\hline
\end{tabular}

\section{Performance Indices (Goodness of Fit Analysis)}

Some methods of goodness of fit analysis are employed here. Mean square error MSE and total deviation TD are two measurements that give an indication of the accuracy of parameter estimation. AL-Fawzan [16] referred to the use of the procedure of MSE and TD.

\subsection{Mean Square Errors (MSE)}

The MSE can be calculated as below

Standard bias, Bias $=\mathrm{E}(\widehat{\beta})-\beta$ and M.S.E $(\widehat{\beta})=\mathrm{E}\left[(\widehat{\beta}-\beta)^{2}\right]$

Standard bias, Bias $=\mathrm{E}(\hat{\gamma})-\gamma$ and M.S.E $(\widehat{\gamma})=\mathrm{E}\left[(\widehat{\gamma}-\gamma)^{2}\right]$

\subsection{Total Derivation (TD)}

The total derivation TD, calculated for each method is as follows

$\mathrm{TD}=\left|\frac{\hat{\gamma}-\gamma}{\gamma}\right|+\left|\frac{\hat{\beta}-\beta}{\beta}\right|$

Where $\gamma$ and $\beta$ are the known parameters, and $\hat{\gamma}$ and $\hat{\beta}$ are the estimated parameters by any method. These techniques are used to measure the variability of parameter estimates for each simulation. These are used to determine the overall "best" parameter estimation method. 


\section{Application}

A simulation study is used in order to compare the performance of the proposed estimation methods. We carried out this comparison by taking the samples of sizes as $\mathrm{n}=20,60$ and 100 with pairs of $(\beta, \gamma)=\{(1,2),(3,2),(4,3)\}$. We generate random samples of different sizes by observing that, if $\mathrm{R}$ is uniform $(0,1)$, then $t_{i}=\beta R_{i}{ }^{1 / \gamma}$ is the random number generator of power function distribution with $(\beta, \gamma)$ parameters. All results are based on 10,000 replications. Such generated data have been used to obtain estimates of the unknown parameters. The results obtained from parameters estimation of the 2-parameters power function distribution using different sample sizes and different values of parameters with mean square error MSE and total deviation TD.

Table 1. MLEs, MMLEs, MEs, and MMEs for the Parameters $\beta$ and $y$ using $n=$ 20

\begin{tabular}{|c|c|c|c|c|c|c|c|}
\hline \multirow{2}{*}{ METHODS } & \multicolumn{2}{|c|}{ True Values } & \multicolumn{2}{|c|}{ Estimated Values } & \multicolumn{2}{|c|}{ M.S.E } & \multirow[t]{2}{*}{ T.D } \\
\hline & $\beta$ & $\gamma$ & $\widehat{\beta}$ & $\hat{\gamma}$ & $\widehat{\beta}$ & $\hat{\gamma}$ & \\
\hline \multirow{3}{*}{ M.L.E } & 1 & 2 & 0.97865 & 2.20073 & 0.00086 & 0.31385 & 0.12172 \\
\hline & 4 & 3 & 3.93356 & 3.26461 & 0.00817 & 0.51884 & 0.10481 \\
\hline & 3 & 2 & 2.91765 & 2.12609 & 0.01228 & 0.20922 & 0.09050 \\
\hline \multirow{4}{*}{ M.M.L.E.1 } & 1 & 2 & 1.13711 & 1.89759 & 0.08873 & 0.52151 & 0.18831 \\
\hline & 4 & 3 & 4.37846 & 2.75087 & 0.61163 & 1.05348 & 0.17766 \\
\hline & 3 & 2 & 3.46170 & 1.81793 & 1.34205 & 0.48311 & 0.24494 \\
\hline & 1 & 2 & 0.97108 & 3.32444 & 0.05472 & 3.89807 & 0.69114 \\
\hline \multirow{2}{*}{ M.M.L.E.2 } & 4 & 3 & 3.94201 & 3.17264 & 0.35571 & 3.45060 & 0.07204 \\
\hline & 3 & 2 & 2.92657 & 3.26293 & 0.59167 & 2.06460 & 0.65594 \\
\hline \multirow{4}{*}{ M.M.L.E.3 } & 1 & 2 & 1.01489 & 2.07033 & 0.00296 & 0.28426 & 0.05006 \\
\hline & 4 & 3 & 4.01024 & 3.13655 & 0.02839 & 0.58332 & 0.04808 \\
\hline & 3 & 2 & 3.02759 & 2.00383 & 0.02948 & 0.20690 & 0.01111 \\
\hline & 1 & 2 & 1.00223 & 2.07033 & 0.00255 & 0.28426 & 0.03740 \\
\hline \multirow{3}{*}{ M.E } & 4 & 3 & 3.98965 & 3.13655 & 0.02789 & 0.58332 & 0.04810 \\
\hline & 3 & 2 & 2.99190 & 2.00383 & 0.03233 & 0.20690 & 0.00462 \\
\hline & 1 & 2 & 1.00223 & 2.07033 & 0.00255 & 0.28426 & 0.03740 \\
\hline \multirow{3}{*}{ M.M.E.1 } & 4 & 3 & 3.98965 & 3.13655 & 0.02789 & 0.58332 & 0.04810 \\
\hline & 3 & 2 & 2.99190 & 2.00383 & 0.03233 & 0.20690 & 0.00462 \\
\hline & 1 & 2 & 1.00223 & 2.07033 & 0.00255 & 0.28426 & 0.03740 \\
\hline \multirow{3}{*}{ M.M.E.2 } & 4 & 3 & 3.98965 & 3.13655 & 0.02789 & 0.58332 & 0.04810 \\
\hline & 3 & 2 & 2.99190 & 2.00383 & 0.03233 & 0.20690 & 0.00462 \\
\hline & 1 & 2 & 1.00223 & 2.07033 & 0.00255 & 0.28426 & 0.03740 \\
\hline \multirow{3}{*}{ M.M.E.3 } & 4 & 3 & 3.98965 & 3.13655 & 0.02789 & 0.58332 & 0.04810 \\
\hline & 3 & 2 & 2.99190 & 2.00383 & 0.03233 & 0.20690 & 0.00462 \\
\hline & 1 & 2 & 1.00223 & 2.07033 & 0.00255 & 0.28426 & 0.03740 \\
\hline \multirow{2}{*}{ M.M.E.4 } & 4 & 3 & 3.98965 & 3.13655 & 0.02789 & 0.58332 & 0.04810 \\
\hline & 3 & 2 & 2.99190 & 2.00383 & 0.03233 & 0.20690 & 0.00462 \\
\hline
\end{tabular}


Table 2. MLEs, MMLEs, MEs, and MMEs for the Parameters $\beta$ and $y$ using $n=$ 60

\begin{tabular}{|c|c|c|c|c|c|c|c|}
\hline \multirow[t]{2}{*}{ METHODS } & \multicolumn{2}{|c|}{ True Values } & \multicolumn{2}{|c|}{ Estimated Values } & \multicolumn{2}{|c|}{ M.S.E } & \multirow[t]{2}{*}{ T.D } \\
\hline & $\beta$ & $\gamma$ & $\widehat{\beta}$ & $\hat{\gamma}$ & $\widehat{\beta}$ & $\hat{\gamma}$ & \\
\hline \multirow{4}{*}{ M.L.E } & 1 & 2 & 0.99087 & 2.03497 & 0.00017 & 0.05089 & 0.02662 \\
\hline & 4 & 3 & 3.97770 & 3.06425 & 0.00093 & 0.13183 & 0.02699 \\
\hline & 3 & 2 & 2.97651 & 2.04607 & 0.00096 & 0.07024 & 0.03086 \\
\hline & 1 & 2 & 1.10741 & 1.88306 & 0.07121 & 0.36356 & 0.16588 \\
\hline \multirow{3}{*}{ M.M.L.E.1 } & 4 & 3 & 4.25862 & 2.77774 & 0.30697 & 0.68814 & 0.13874 \\
\hline & 3 & 2 & 3.34599 & 1.83435 & 0.50906 & 0.33646 & 0.19816 \\
\hline & 1 & 2 & 0.95834 & 2.64694 & 0.02035 & 4.39336 & 0.36513 \\
\hline \multirow{3}{*}{ M.M.L.E.2 } & 4 & 3 & 3.96967 & 3.56070 & 0.16228 & 2.65240 & 0.19448 \\
\hline & 3 & 2 & 3.04299 & 2.17227 & 0.18936 & 0.63329 & 0.10047 \\
\hline & 1 & 2 & 0.99994 & 2.00973 & 0.00085 & 0.06592 & 0.00492 \\
\hline \multirow{3}{*}{ M.M.L.E.3 } & 4 & 3 & 3.99752 & 3.03784 & 0.00903 & 0.17865 & 0.01323 \\
\hline & 3 & 2 & 3.02187 & 1.99269 & 0.00861 & 0.07407 & 0.01095 \\
\hline & 1 & 2 & 0.99495 & 2.00973 & 0.00090 & 0.06592 & 0.00991 \\
\hline \multirow{3}{*}{ M.E } & 4 & 3 & 3.99149 & 3.03784 & 0.00973 & 0.17865 & 0.01474 \\
\hline & 3 & 2 & 3.01301 & 1.99269 & 0.00945 & 0.07407 & 0.00799 \\
\hline & 1 & 2 & 0.99495 & 2.00973 & 0.00090 & 0.06592 & 0.00991 \\
\hline \multirow{3}{*}{ M.M.E.1 } & 4 & 3 & 3.99149 & 3.03784 & 0.00973 & 0.17865 & 0.01474 \\
\hline & 3 & 2 & 3.01301 & 1.99269 & 0.00945 & 0.07407 & 0.00799 \\
\hline & 1 & 2 & 0.99495 & 2.00973 & 0.00090 & 0.06592 & 0.00991 \\
\hline \multirow{3}{*}{ M.M.E.2 } & 4 & 3 & 3.99149 & 3.03784 & 0.00973 & 0.17865 & 0.01474 \\
\hline & 3 & 2 & 3.01301 & 1.99269 & 0.00945 & 0.07407 & 0.00799 \\
\hline & 1 & 2 & 0.99495 & 2.00973 & 0.00090 & 0.06592 & 0.00991 \\
\hline \multirow{3}{*}{ M.M.E.3 } & 4 & 3 & 3.99149 & 3.03784 & 0.00973 & 0.17865 & 0.01474 \\
\hline & 3 & 2 & 3.01301 & 1.99269 & 0.00945 & 0.07407 & 0.00799 \\
\hline & 1 & 2 & 0.99495 & 2.00973 & 0.00090 & 0.06592 & 0.00991 \\
\hline \multirow[t]{2}{*}{ M.M.E.4 } & 4 & 3 & 3.99149 & 3.03784 & 0.00973 & 0.17865 & 0.01474 \\
\hline & 3 & 2 & 3.01301 & 1.99269 & 0.00945 & 0.07407 & 0.00799 \\
\hline
\end{tabular}

Table 3. MLEs, MMLEs, MEs, and MMEs for the Parameters $\beta$ and $y$ using $n=$ 100

\begin{tabular}{cccccccc}
\hline METHODS & \multicolumn{2}{c}{ True Values } & \multicolumn{2}{c}{ Estimated Values } & \multicolumn{2}{c}{ M.S.E } & T.D \\
& $\beta$ & $\gamma$ & $\hat{\beta}$ & $\hat{\gamma}$ & $\hat{\beta}$ & $\hat{\gamma}$ & \\
\hline \multirow{3}{*}{ M.L.E } & 1 & 2 & 0.99534 & 2.04891 & 0.00004 & 0.04073 & 0.02912 \\
& 4 & 3 & 3.98666 & 3.04785 & 0.00032 & 0.10940 & 0.01929 \\
& 3 & 2 & 2.98528 & 2.04407 & 0.00041 & 0.03943 & 0.02694 \\
& 1 & 2 & 1.08190 & 1.90833 & 0.03547 & 0.29046 & 0.12774 \\
M.M.L.E.1 & 4 & 3 & 4.26553 & 2.78083 & 0.33442 & 0.70623 & 0.13944 \\
& 3 & 2 & 3.26940 & 1.85885 & 0.34846 & 0.22862 & 0.16037 \\
\hline
\end{tabular}




\begin{tabular}{cccccccc}
\hline & 1 & 2 & 0.98418 & 2.29333 & 0.01339 & 0.65291 & 0.16249 \\
M.M.L.E.2 & 4 & 3 & 3.98510 & 3.25239 & 0.09420 & 0.90408 & 0.08786 \\
& 3 & 2 & 3.04372 & 2.09899 & 0.11714 & 0.36161 & 0.06407 \\
M.M.L.E.3 & 1 & 2 & 1.00085 & 2.03064 & 0.00045 & 0.04511 & 0.01617 \\
& 3 & 3 & 3.99879 & 3.03322 & 0.00458 & 0.14713 & 0.01138 \\
& 1 & 2 & 3.01057 & 2.01212 & 0.00437 & 0.03781 & 0.00959 \\
M.E & 4 & 2 & 0.99824 & 2.03064 & 0.00052 & 0.04511 & 0.01708 \\
& 3 & 2 & 3.99683 & 3.03322 & 0.00553 & 0.14713 & 0.01187 \\
M.M.E.1 & 1 & 2 & 0.99824 & 2.03064 & 0.00052 & 0.04511 & 0.01708 \\
& 4 & 3 & 3.99683 & 3.03322 & 0.00553 & 0.14713 & 0.01187 \\
M.M.E.2 & 1 & 2 & 3.00647 & 2.01212 & 0.00482 & 0.03781 & 0.00822 \\
& 4 & 3 & 0.99824 & 2.03064 & 0.00052 & 0.04511 & 0.01708 \\
& 1 & 2 & 3.99683 & 3.03322 & 0.00553 & 0.14713 & 0.01187 \\
M.M.E.3 & 4 & 3 & 0.99824 & 2.03064 & 0.00052 & 0.04511 & 0.01708 \\
& 3 & 2 & 3.00647 & 2.01212 & 0.00482 & 0.03781 & 0.00822 \\
M.M.E.4 & 1 & 2 & 0.99824 & 2.03064 & 0.00052 & 0.04511 & 0.01708 \\
& 4 & 3 & 3.99683 & 3.03322 & 0.00553 & 0.14713 & 0.01187 \\
& 3 & 2 & 3.00647 & 2.01212 & 0.00482 & 0.03781 & 0.00822 \\
\hline
\end{tabular}

Table 4. PEs, and MPEs for the parameters $\beta$ and $Y$ using $\mathbf{n}=20$

\begin{tabular}{|c|c|c|c|c|c|c|c|}
\hline \multirow{2}{*}{ METHODS } & \multicolumn{2}{|c|}{ True Values } & \multicolumn{2}{|c|}{ Estimated Values } & \multicolumn{2}{|c|}{ M.S.E } & \multirow[t]{2}{*}{ T.D } \\
\hline & $\beta$ & $\gamma$ & $\widehat{\beta}$ & $\hat{\gamma}$ & $\widehat{\beta}$ & $\hat{\gamma}$ & \\
\hline \multirow{3}{*}{ P.E $E_{(5,95)}$} & 1 & 2 & 0.96331 & 1.91461 & 0.00084 & 0.33627 & 0.04911 \\
\hline & 4 & 3 & 3.93834 & 2.68987 & 0.00658 & 0.79654 & 0.09988 \\
\hline & 3 & 2 & 3.05117 & 2.51692 & 0.00988 & 0.33698 & 0.09633 \\
\hline \multirow{3}{*}{ M.P.E.1 $(5,95)$} & 1 & 2 & 1.00641 & 1.94920 & 0.00054 & 0.34987 & 0.05270 \\
\hline & 4 & 3 & 4.00928 & 2.79071 & 0.00411 & 0.64841 & 0.07208 \\
\hline & 3 & 2 & 3.00378 & 1.82828 & 0.00599 & 0.28848 & 0.08712 \\
\hline \multirow{3}{*}{ M.P.E.2 $(5,95)$} & 1 & 2 & 0.96288 & 1.98285 & 0.00987 & 0.31587 & 0.06988 \\
\hline & 4 & 3 & 3.90627 & 4.70869 & 0.00895 & 0.87598 & 1.00258 \\
\hline & 3 & 2 & 3.05623 & 2.32768 & 1.02356 & 0.44569 & 0.99987 \\
\hline \multirow{3}{*}{ M.P.E.3 $(5,95)$} & 1 & 2 & 0.95553 & 2.81742 & 0.02568 & 0.69874 & 0.08974 \\
\hline & 4 & 3 & 3.99683 & 1.51702 & 0.36547 & 0.89547 & 1.12547 \\
\hline & 3 & 2 & 3.05095 & 2.52579 & 0.12458 & 1.02365 & 0.09999 \\
\hline \multirow{4}{*}{ P.E $E_{(25,75)}$} & 1 & 2 & 1.02118 & 1.94920 & 0.00676 & 0.39974 & 0.03740 \\
\hline & 4 & 3 & 4.03348 & 2.68987 & 0.05474 & 0.74673 & 0.01243 \\
\hline & 3 & 2 & 3.06322 & 2.51692 & 0.05602 & 0.50222 & 0.01792 \\
\hline & 1 & 2 & 1.01467 & 2.02845 & 0.00255 & 0.28426 & 0.02889 \\
\hline M.P.E.1 $(25,75)$ & 4 & 3 & 4.14214 & 2.98782 & 0.02789 & 0.58332 & 0.04810 \\
\hline
\end{tabular}




\begin{tabular}{lccccccc}
\hline & 3 & 2 & 3.01493 & 2.02589 & 0.03233 & 0.20690 & 0.00462 \\
& 1 & 2 & 1.06012 & 1.55491 & 0.00255 & 0.28426 & 0.03740 \\
M.P.E.2 $_{(25,75)}$ & 4 & 3 & 3.99252 & 4.10038 & 0.02789 & 0.58332 & 0.04810 \\
& 3 & 2 & 3.11731 & 2.18263 & 0.03233 & 0.20690 & 0.00462 \\
& 1 & 2 & 0.97791 & 2.75832 & 0.00255 & 0.28426 & 0.03740 \\
M.P.E.3 $_{(25,75)}$ & 4 & 3 & 4.55513 & 1.42423 & 0.02789 & 0.58332 & 0.04810 \\
& 3 & 2 & 3.06314 & 2.51746 & 0.03233 & 0.20690 & 0.00462 \\
\hline
\end{tabular}

Table 5. PEs, and MPEs for the Parameters $\beta$ and $y$ using $\mathbf{n}=\mathbf{6 0}$

\begin{tabular}{|c|c|c|c|c|c|c|c|}
\hline \multirow{2}{*}{ METHODS } & \multicolumn{2}{|c|}{ True } & \multicolumn{2}{|c|}{ Estimated Values } & \multicolumn{2}{|c|}{ M.S.E } & \multirow[t]{2}{*}{ T.D } \\
\hline & $\beta$ & $\gamma$ & $\widehat{\beta}$ & $\hat{\gamma}$ & $\widehat{\beta}$ & $\hat{\gamma}$ & \\
\hline \multirow{3}{*}{ P.E $E_{(5,95)}$} & 1 & 2 & 1.00362 & 2.02094 & 0.07121 & 0.13448 & 0.01197 \\
\hline & 4 & 3 & 4.01174 & 3.36567 & 0.30697 & 0.68814 & 0.13874 \\
\hline & 3 & 2 & 3.03379 & 1.67547 & 0.50906 & 0.33646 & 0.19816 \\
\hline \multirow{3}{*}{ M.P.E. $1_{(5,95)}$} & 1 & 2 & 1.00151 & 2.11504 & 0.00023 & 0.36356 & 0.16588 \\
\hline & 4 & 3 & 4.00272 & 2.94508 & 0.00150 & 0.29377 & 0.01899 \\
\hline & 3 & 2 & 3.00663 & 1.93209 & 0.00179 & 0.13334 & 0.03616 \\
\hline \multirow{3}{*}{ M.P.E.2 $(5,95)$} & 1 & 2 & 1.01130 & 1.60909 & 0.02035 & 4.39336 & 0.36513 \\
\hline & 4 & 3 & 4.04036 & 2.29499 & 0.16228 & 2.65240 & 0.19448 \\
\hline & 3 & 2 & 3.02366 & 1.88107 & 0.18936 & 0.63329 & 0.10047 \\
\hline \multirow{3}{*}{ M.P.E.3 $(5,95)$} & 1 & 2 & 1.00519 & 1.98730 & 0.00085 & 0.06592 & 0.00492 \\
\hline & 4 & 3 & 4.01585 & 3.15379 & 0.00903 & 0.17865 & 0.01323 \\
\hline & 3 & 2 & 3.04060 & 1.56118 & 0.00861 & 0.33936 & 0.01095 \\
\hline \multirow{4}{*}{ P. $E_{(25,75)}$} & 1 & 2 & 0.98780 & 1.94909 & 0.00221 & 0.16304 & 0.02936 \\
\hline & 4 & 3 & 3.93727 & 3.36567 & 0.01663 & 0.33936 & 0.01474 \\
\hline & 3 & 2 & 3.18543 & 1.67547 & 0.02242 & 0.16304 & 0.00799 \\
\hline & 1 & 2 & 1.00390 & 2.11504 & 0.00090 & 0.06592 & 0.00991 \\
\hline \multirow{2}{*}{ M.P.E.1 $1_{(25,75)}$} & 4 & 3 & 3.99727 & 3.03105 & 0.00973 & 0.17865 & 0.01103 \\
\hline & 3 & 2 & 3.01955 & 2.01986 & 0.00945 & 0.07407 & 0.01645 \\
\hline \multirow{4}{*}{ M.P.E.2 $2_{(25,75)}$} & 1 & 2 & 1.04515 & 1.49485 & 0.00090 & 0.06592 & 0.00991 \\
\hline & 4 & 3 & 4.13844 & 2.12615 & 0.00973 & 0.17865 & 0.01474 \\
\hline & 3 & 2 & 3.20107 & 1.62901 & 0.00945 & 0.07407 & 0.00799 \\
\hline & 1 & 2 & 0.99583 & 1.99633 & 0.00090 & 0.06592 & 0.00991 \\
\hline \multirow{2}{*}{ M.P.E.3 $(25,75)$} & 4 & 3 & 3.95577 & 3.19068 & 0.00973 & 0.17865 & 0.01474 \\
\hline & 3 & 2 & 3.24010 & 1.52439 & 0.00945 & 0.07407 & 0.00799 \\
\hline
\end{tabular}

Table 6. PEs, and MPEs for the Parameters $\beta$ and $y$ using $n=100$

\begin{tabular}{cccccccc}
\hline \multirow{2}{*}{ METHODS } & \multicolumn{2}{c}{ True Values } & \multicolumn{2}{c}{ Estimated Values } & \multicolumn{2}{c}{ M.S.E } & \multirow{2}{*}{ T.D } \\
& $\beta$ & $\gamma$ & $\hat{\beta}$ & $\hat{\gamma}$ & $\hat{\beta}$ & $\hat{\gamma}$ & \\
\hline & 1 & 2 & 0.99228 & 2.40349 & 0.03547 & 0.29046 & 0.12774 \\
P.E $_{(5,95)}$ & 4 & 3 & 3.95831 & 3.34118 & 0.33442 & 0.70623 & 0.13944 \\
\hline
\end{tabular}




\begin{tabular}{|c|c|c|c|c|c|c|c|}
\hline \multirow{4}{*}{ M.P.E.1 $(5,95)$} & 3 & 2 & 3.02558 & 1.90685 & 0.34846 & 0.22862 & 0.16037 \\
\hline & 1 & 2 & 1.00037 & 2.00096 & 0.00016 & 0.09768 & 0.00085 \\
\hline & 4 & 3 & 4.00047 & 2.94087 & 0.00096 & 0.19645 & 0.01983 \\
\hline & 3 & 2 & 3.00697 & 1.97611 & 0.00133 & 0.08334 & 0.01427 \\
\hline \multirow{3}{*}{ M.P.E.2 $(5,95)$} & 1 & 2 & 0.99389 & 2.22899 & 0.01339 & 0.65291 & 0.16249 \\
\hline & 4 & 3 & 3.94751 & 4.06448 & 0.09420 & 0.90408 & 0.08786 \\
\hline & 3 & 2 & 3.02276 & 1.97528 & 0.11714 & 0.36161 & 0.06407 \\
\hline \multirow{3}{*}{ M.P.E.3 $(5,95)$} & 1 & 2 & 0.98821 & 2.96813 & 0.00045 & 0.04511 & 0.01617 \\
\hline & 4 & 3 & 3.99627 & 2.06027 & 0.00458 & 0.14713 & 0.01138 \\
\hline & 3 & 2 & 3.03948 & 1.62927 & 0.00437 & 0.03781 & 0.00959 \\
\hline \multirow{4}{*}{ P.E $(25,75)$} & 1 & 2 & 0.98889 & 2.40349 & 0.00052 & 0.04511 & 0.01708 \\
\hline & 4 & 3 & 4.01869 & 3.34118 & 0.00553 & 0.14713 & 0.01187 \\
\hline & 3 & 2 & 3.05026 & 1.90685 & 0.00482 & 0.03781 & 0.00822 \\
\hline & 1 & 2 & 1.00088 & 2.01462 & 0.00112 & 0.07766 & 0.00818 \\
\hline \multirow{3}{*}{ M.P.E.1 $1_{(25,75)}$} & 4 & 3 & 3.99190 & 3.06999 & 0.00928 & 0.27249 & 0.02535 \\
\hline & 3 & 2 & 3.00093 & 2.03073 & 0.01008 & 0.06768 & 0.01567 \\
\hline & 1 & 2 & 1.00128 & 2.17713 & 0.00052 & 0.04511 & 0.01708 \\
\hline \multirow{3}{*}{ M.P.E.2 $(25,75)$} & 4 & 3 & 3.96475 & 3.96322 & 0.00553 & 0.14713 & 0.01187 \\
\hline & 3 & 2 & 3.04261 & 1.93913 & 0.00482 & 0.03781 & 0.00822 \\
\hline & 1 & 2 & 0.96521 & 3.01376 & 0.00052 & 0.04511 & 0.01708 \\
\hline \multirow{2}{*}{ M.P.E.3 $(25,75)$} & 4 & 3 & 4.25638 & 2.00387 & 0.00553 & 0.14713 & 0.01187 \\
\hline & 3 & 2 & 3.13577 & 1.61152 & 0.00482 & 0.03781 & 0.00822 \\
\hline
\end{tabular}

\section{Results And Conclusions}

All the results are listed in tables 1 to 6 . A Monte Carlo simulation study shows that if we compare the M.L.E, M.E and their modifications, we find that for small sample size, their modified estimators produce better results. But as the sample size increases, the M.L.E provides the best results. M.E does not show any reliable results. It is very interesting that the result of M.E and its modifications are equal but their formulae are different.

The comparison of Percentile estimators (P.E) and its modifications suggests that for small sample size the Modified percentile estimators (M.P.E) produce the best estimates for $\beta$ and $\gamma$ with some exceptions. However, as the sample size increases the M.P.E.1 (First Modified Percentile Estimator) provides the best estimators (the method which has least M.S.E).

Among all the Percentile estimators and their modifications, $\mathrm{P}_{(5,25)}$ produces the best results, which shows that larger data produce better results. The best estimator among all the Percentile estimators and Modified percentile estimators is M.P.E.1 $1_{(5,95)}$

Finally, we recommend using M.P.E.1 and M.L.E modifications methods for small sample size and M.L.E for larger sample size for the estimation of the parameters of the power function distribution.

\section{References}

[1] M. Ahsanullah, "Estimation of the location and scale parameters of a Power function distribution by linear functions of order statistics", Communications in Statistics, vol. 5, (1974), pp. 463-467.

[2] M. Ahsanullah, "Estimation of the parameters of a Power function distribution by record values", Pak. J. Statistics, vol. 5, no. 2(a), (1989), pp. 189-194. 
[3] M. Ahmad and A. M. Al-Marzoug, "Estimation of parameters of Burr probability models using fractional moments”, Pak. J. Statist, vol. 1, no. 1, (1985), pp. 67-77.

[4] A. C. Cohen and B. J. Whitten, "Estimation in the three parameters lognormal distribution", Journal of the American Statistical Association, vol. 75, (1980), pp. 399-404.

[5] A. C. Cohen, "Modified moment and maximum likelihood estimators for parameters of three parameters gamma distribution”, Communication in Statistics. vol. 11, (1982), pp. 197-216.

[6] A. C. Cohen, "Modified moment and maximum likelihood estimators for parameters of three parameters Weibull distribution”, Communication in Statistics, vol. 11, (1982), pp. 2631-2656.

[7] S. D. Dubey, "Some percentile estimators for Weibull parameters”, Technometrics, vol. 9, (1967), pp. 119129.

[8] N. L. Johnson and S. Kotz, "Distributions in Statistics continuous univariate distributions”, New York, John Wiley, (1970).

[9] K. Kapadia, "Sample size required to estimate a parameter in the Power function distribution”, Madrid, vol. 29, (1978).

[10] S. B. Kang and C. S. Young, "Estimation of the Parameters in a Pareto Distribution by Jackknife and Bootstrap Methods”, Journal of Information and optimization Sci, vol. 18, (1997), pp. 289-300.

[11] S. Lalitha, M. Anand, "Modified Maximum Likelihood Estimators for Rayleigh Distribution", Commun. Statist, Theory and Method, vol. 25, no. 2, (1996), pp. 389-401.

[12] T. Lwin, "Estimation of the tail of Paretian law", Skandinavisk aktuarietiidskrift, vol. 55, (1972), pp. 170178.

[13] H. J. Malik, "Exact moments of order statistics for a power function distribution", Skandinavisk aktuarietiidskrift, vol. 50, (1967), pp. 64-69.

[14] B. Meniconi, “The power function distribution: A useful and simple distribution to asses' electrical component reliability”, Microelectron.Reliab, vol. 36, no. 9, (1995), pp. 1207-1212.

[15] M. A. AL-Fawzan, “Algorithms for Estimating the Parameters of the Weibull Distribution”, King AbdulAziz City for Science and Technology, Riyadh, Saudi Arabia, (2000).

[16] S. Munawar and M. Farooq, "Bayesian parameters estimation of hybrid censored power function distribution under different loss functions”, $9^{\text {th }}$ International Conference on Statistical Sciences Lahore, Pakistan. vol. 22, (2012), pp. 331-340.

[17] M. Neil, "Estimation of Weibull parameters from common percentiles”, Journal of applied Statistics, vol. 32, (2005), pp. 17-24.

[18] B. S. Pablo and E. R. Bruce, "Model Parameter Estimation using Least Squares”, Water Res. vol. 26, no. 6, (1992), pp. 789-796.

[19] T. F. Peter, "Huber- Sense Robust M-Estimation of a Scale Parameter, with Application to the Exponential Distribution”, JASA, vol. 74, (1974), pp. 147-152.

[20] P. R. Rider, "Distribution of the product, quotient of maximum values in samples from a power function population”, Journal of the American Statistical Association, vol. 59, (1964), pp. 877-880.

[21] Rafiq, "Estimation of parameters of the Gamma distribution by the method of fractional moments", Pak. J. Statist, vol. 12, no. 3, (1996), pp. 265-274.

[22] Rafiq, "Estimation of parameters of the Weibull distribution by the method of fractional moments”, Pak. J. Statist, vol. 15, no. 2, (1999), pp. 91-96.

[23] A. S. Samia and M. M. Mohammed, "Modified Moment Estimators for three parameters Pareto distribution”, ISSR, Cairo University, vol. 28, no. 2, (1993).

[24] S. Zarrin, S. Saxena and K. Mustafa, "Reliability computation and Bayesian Analysis of system reliability of Power function distribution”, International Journal of Advance in Engineering, Science and Technology, vol. 2, no. 4, (2013), pp. 76-86. 